\title{
A!
}

This is an electronic reprint of the original article.

This reprint may differ from the original in pagination and typographic detail.

Wang, Yuzhou; Masoodian, Masood

\section{Designing Visual Tools to Facilitate Human-Centered Design}

\section{Published in:}

Proceedings of the Working Conference on Advanced Visual Interfaces

DOI:

$10.1145 / 3399715.3399936$

Published: 28/09/2020

Document Version

Peer reviewed version

Please cite the original version:

Wang, Y., \& Masoodian, M. (2020). Designing Visual Tools to Facilitate Human-Centered Design. In G. Tortora, G. Vitiello, \& M. Winckler (Eds.), Proceedings of the Working Conference on Advanced Visual Interfaces: AVI'20 (pp. 1-3). [81] ACM. https://doi.org/10.1145/3399715.3399936

This material is protected by copyright and other intellectual property rights, and duplication or sale of all or part of any of the repository collections is not permitted, except that material may be duplicated by you for your research use or educational purposes in electronic or print form. You must obtain permission for any other use. Electronic or print copies may not be offered, whether for sale or otherwise to anyone who is not an authorised user. 


\section{Designing Visual Tools to Facilitate Human-Centered Design}

\author{
Yuzhou Wang \\ School of Arts, Design and Architecture \\ Aalto University \\ Espoo, Finland \\ yuzhou.wang@aalto.fi
}

\author{
Masood Masoodian \\ School of Arts, Design and Architecture \\ Aalto University \\ Espoo, Finland \\ masood.masoodian@aalto.fi
}

\begin{abstract}
Human-Centred Design (HCD) relies on the use of many methods (e.g. interviews, observations) originating from other disciplines such as social sciences (e.g. ethnography). Such methods often rely on the use of visual tools (e.g. photographs and illustrations) to better facilitate the involvement of the participants in the design process. Most HCD practitioners, however, do not have the necessary visual design skills, and as such, need to work with visual communication designers to co-create visual tools to support their design projects. In this poster, we present a multidisciplinary approach to guide the process of co-creating such visual tools.
\end{abstract}

\section{KEYWORDS}

Visual material, visual design, visual methods, human-centered design, co-design, qualitative research.

\section{INTRODUCTION}

Visual tools are often used in qualitative research methods [5]for instance, in ethnographic research [1] to evoke comments and feedback from the research participants [6]. Many of these methods have also been adapted and commonly used in Human-Centered Design (HCD). For example, methods based on interviews and focus groups are used to gather user requirements during the discover phase of the Double Diamond design process [2]. Similarly, IDEO [4], for instance, promotes the use of visual tools by design research participants as part of a self-documentation method.

Most HCD practitioners, however, do not have the necessary Visual Communication (VC) design skills to create suitable visual tools for use in their design projects, and therefore, need to work with VC designers to create such tools. Furthermore, generally it is not simply the case that HCD practitioners can ask VC designers to produce the required visual tools on their own-due to the fact the VC designers may not fully understand all the HCD requirements.

Therefore, in this poster, we propose and discuss-using a case example-a co-creation process involving HCD practitioners and $\mathrm{VC}$ designers to produce visual tools for use in HCD projects.

\section{TYPOLOGY OF VISUAL TOOLS}

Since a wide range of visual tools are used in qualitative methods, it can be challenging to determine what type of tools to use in a project. Glegg [3] proposes a typology that arranges visual tools into five categories according to their purpose: to 1) enable communication, 2) represent data, 3) facilitate relationships, 4) enhance data quality and validity, and 5) affect change. While this typology is also useful for HCD purposes, there are a number of points to keep in mind. Firstly, there are some overlapping functions for visual tools in Glegg's typology, especially between the categories 1 and 3. For instance, while the function "develop rapport" is included in category 3 , it is also important for improving communication (i.e. category 1). Secondly, Glegg's suggestion that applying the typology relies solely on the researcher's perspective, ignores the fact that, for our purpose, HCD practitioners often work within multidisciplinary teams-not to mention that here we are proposing the need for them to collaborate with VC designers as well.

With these HCD needs in mind, we propose adapting and using Glegg's typology in deciding the type of visual tools required for specific design methods used in a project. For example, in the case of interviews, visual tools are needed for facilitating communication, engaging participants, eliciting comments and fostering ideas.

\section{CO-DESIGN OF VISUAL TOOLS}

Figure 1 depicts the co-design process involving HCD practitioners and VC designers, who work together on: 1) brainstorming ideas, 2) creating visual prototypes, and 3) iterating, testing and refining prototypes to produce the final visual tools. Both parties also advise each other based on their own expertise. For instance, HCD practitioners, who might have a deeper understanding of the cultural context of their design project, can advise $\mathrm{VC}$ designers to produce more culturally appropriate visual tools. VC designers, on the other hand, can for instance recommend a particular visual style or content which are more suitable for a specific cultural setting.

To support such interactions, we propose a 4-stage co-design process, which we discuss here using our case example of creating visual tools to support conducting field interviews in Bihar, India.

(1) Define the requirements of the visual tools: The visual tools were needed for interviews exploring the lives of females (aged 10-22). Two important factors had to considered: 1) the participants did not widely use the internet or digital 


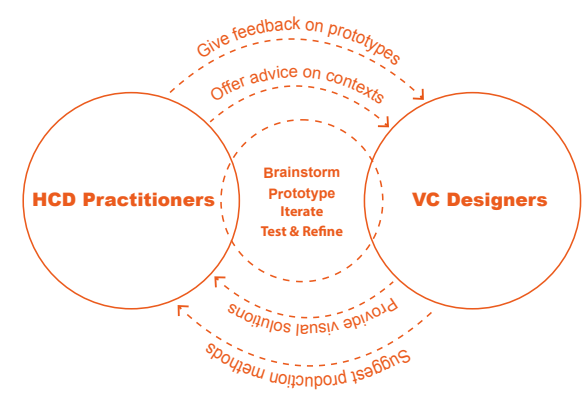

Figure 1: The proposed iterative co-creation process.

devices-so the visual tools needed to be physical and tangible, and 2) the interviews would include sensitive topics (e.g. puberty and sex)-so the visual tools had to minimize any negative influences. Based on these needs, the concept of paper dolls was chosen as the basis for the visual tools.

(2) Choose an appropriate visual style: The level of visual abstraction was considered using McCloud's [7] proposed triangular diagram, along its two dimensions of: 1) Iconic abstraction which leads to "Meaning", and 2) Pure abstraction which leads to "The Picture Plane". It was decided that the visual representation of the paper dolls should follow the axis of iconic abstraction, because the visual material must be understood by both the HCD practitioners and research participants sharing a common meaning. Figure 2 shows an example of the process of image abstraction which was followed to create an illustration based on the photograph of a Hindu temple. The process consists of three steps: 1) summarize shape or configuration (e.g. the temple is a structure with two towers and a chamber with two doors), 2) remove irrelevant details (e.g. decorative elements are removed), and 3) preserve distinctive features (e.g. the unique shape of the door, and the five layers of the towers).

(3) Create visual prototypes: Several prototypes were created, ranging from low-fidelity prototypes (e.g. collage of photographs), to high-fidelity prototypes produced after the completion of early illustrations. During this process, the team agreed on some key elements of the paper dolls and their possible usage scenarios. Figure 3 shows different components of the final visual tools: a paper doll, different outfits, and multiple environment cards. The paper doll is the main component and can represent a persona based on real-life

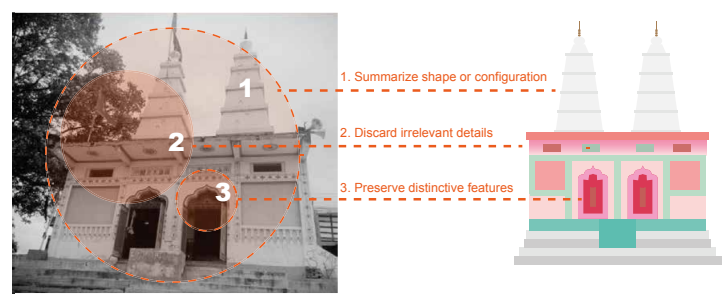

Figure 2: The three steps of image abstraction.

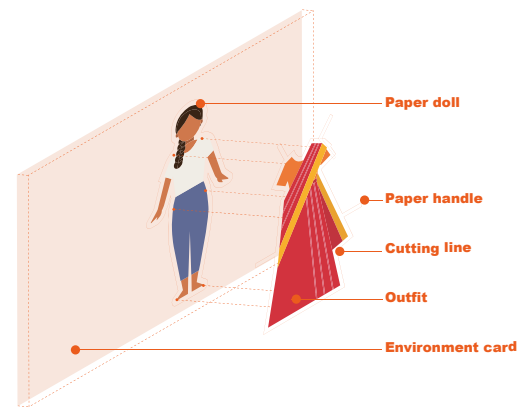

Figure 3: Components of the paper dolls visual tools.

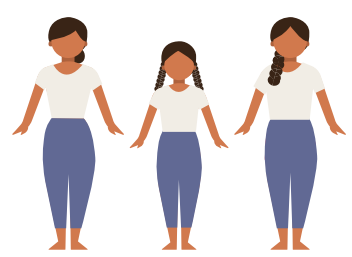

Figure 4: Three different hair styles of the paper dolls.

settings of the design participants-and by building narratives around the paper doll, participants can associate their experiences with the persona of the doll and share their own stories. Different sets of clothes (e.g. saree, school uniform) can be used to represent different transition points in the life course of the doll's persona. The outfits also have paper handles to attach them to the doll. The paper doll, along with an outfit, can be placed on different environments (e.g. a temple), which allows, for instance, HCD practitioners to construct more specific narratives and ask focused questions.

(4) Iterate, test and refine: Through the iterative process of testing and refining, it was realized that a single doll representation could not be used for all the different age groups and life stages. This is because females in India have distinct hairstyles in different stages of their lives or different occasions. It was, therefore, decided to create dolls with three different hair styles, as shown in Figure 4. It also became clear through testing that various supplementary characters (e.g. teacher, health worker) were needed. The other visual features of these professional characters were kept unspecified, to allow associating different meanings to them.

\section{CONCLUSION}

We have found the proposed co-design process valuable in creating the visual tools needed as part of the case example presented here. The final visual tools have been produced and recently used successfully in field interviews with individuals and groups in India.

\section{ACKNOWLEDGMENTS}

Yuzhou Wang was also employed at Scope Impact Oy (Finland) and worked as a visual designer creating the paper dolls visual tools. 


\section{REFERENCES}

[1] Marcus Banks. 2018. Using Visual Data in Qualitative Research (2nd ed.). SAGE Publications Ltd, London.

[2] Design Council. 2020. What is the framework for innovation? Design Council's evolved Double Diamond. https://www.designcouncil.org.uk

[3] Stephanie M. N. Glegg. 2019. Facilitating Interviews in Qualitative Research With Visual Tools: A Typology. Qualitative Health Research 29, 2 (2019), 301-310. https://doi.org/10.1177/1049732318786485
[4] IDEO.org. 2015. The Field Guide to Human-Centered Design (1st ed.). IDEO.org

[5] Davison Jane, McLean Christine, and Warren Samantha. 2015. Looking back: ten years of visual qualitative research. Qualitative Research in Organizations and Management: An International fournal 10, 4 (2015), 355-359. https://doi.org/10. 1108/QROM-07-2015-1311

[6] Victor Jupp. 2006. The SAGE Dictionary of Social Research Methods. SAGE Publications Ltd, London.

[7] Scott McCloud. 1994. Understanding Comics: The Invisible Art. William Morrow Paperbacks, Northampton, MA. 